\title{
Surgical Treatment Strategy with Combined Cardiopulmonary Bypass for Renal Cell Carcinoma with Tumor Embolism Developed in Inferior Vena Cava
}

\author{
Tomohiro Imazuru, MD, PhD, Masateru Uchiyama, MD, PhD, Tomoki Shimokawa, MD, PhD \\ Department of Cardiovascular Surgery, Teikyo University, Tokyo, Japan
}

\section{ABSTRACT}

Objective: Renal cell carcinoma (RCC) with tumor thrombus in the inferior vena cava (IVC) presents surgeons with a technical intraoperative challenge because of the need for aggressive surgical management. In this study, we describe our method for surgical management with cardiopulmonary bypass $(\mathrm{CPB})$ and investigate the long-term outcomes of RCC patients with and without CPB.

Methods: Fifteen patients with RCC underwent nephrectomy and IVC thrombectomy from May 2011 to December 2017. We retrospectively reviewed and analyzed the clinical course of all patients. Novick classification was used to assess the level of tumor thrombus extension into the IVC. Patient characteristics, surgical procedures, and postoperative outcome data in both groups were collected.

Results: Twelve patients were male and 3 were female, with an average age of $62.9 \pm 10.9$ years (range 46 to 82 ). The average operative times were $824 \pm 335$ minutes in the patients with $\mathrm{CPB}$ and $646 \pm 162$ minutes in those without CPB $(P=.17)$. The average amount of intraoperative bleeding was $2125 \pm 1315 \mathrm{ml}$ in the patients with $\mathrm{CPB}$ and $3333 \pm$ $1431 \mathrm{ml}$ in those without $\mathrm{CPB}(P=.14)$. The same tendency was observed in patients of Novick levels 3 and 4 . The mean observation period was 1061.4 days. No 30-day mortality was noted. There was no significant difference in all-cause survival between the patients with $\mathrm{CPB}$ and those without.

Conclusions: We conclude that surgical management with $\mathrm{CPB}$ and circulatory arrest may be a viable and safe method of treatment for RCC patients.

\section{INTRODUCTION}

Renal cell carcinoma (RCC), the most common tumor within urogenital malignant tumors, accounts for $3 \%$ of all solid cancers [Chan 2001]. Overall, RCC incidence rates have continued to increase (on average $2.5 \%$ per year), which may be partly attributed to the advancement of image diagnosis devices and methods [Chowdhury 2007]. Moreover, 20\% to $30 \%$ of RCC patients have developed metastases at the time

Received September 23, 2019; accepted October 23, 2019.

Correspondence: Tomohiro Imazuru, $M D, P h D$, Department of Cardiovascular Surgery, Teikyo University, 2-11-1 Kaga, Itabasbi-ku, Tokyo, 173-8605, Japan; 81-3-3964-1211 (e-mail: imazuru@med.teikyo-u.ac.jp). of diagnosis or after surgical resection [Janzen 2003]. Generally speaking, RCC tends to invade the renal venous system and form a tumor thrombus extension. Approximately $4 \%$ to $10 \%$ of patients with RCC have tumor thrombus invading the inferior vena cava (IVC), and 1\% have tumor thrombus extending into the right atrium, with poor prognosis [Ciancio 2010; Marshall 1988]. However, some studies have demonstrated that 5-year survival rates for patients with tumor thrombi in the absence of metastases after aggressive surgical management were $32 \%$ to $68 \%$, and that the level of thrombus was independent of the prognosis [Jemal 2010; Staehler 2000; Bachmann 2005].

In almost all patients with RCC and thrombus extension, radical nephrectomy with tumor thrombectomy is performed. It is known, however, that higher-level tumor thrombectomy requires highly invasive surgery and precise surgical management, which may be associated with high preoperative mortality and complication rates. In particular, increased complications in patients with level 3 and 4 thrombus extension seem to be due to invasive surgical procedures such as cardiopulmonary bypass (CPB) and veno-venous bypass [Ali 2013; Kaplan 2002; Lawindy 2012; Karnes 2008]. Precise excision of an intrahepatic caval tumor (level 3), including the approach to the liver, is performed in cooperation with thoracic surgeons and hepatobiliary pancreatic surgeons [Ciancio 2007; Ciancio 2005]. In cases of suprahepatic thrombus (level 4), $\mathrm{CPB}$ and circulatory arrest for complete removal of tumor thrombi are permitted; however, there is controversy regarding appropriate intraoperative management in patients with level 1 to 3 thrombi and whether methods such as CPB are appropriate [Blute 2004; Dominik 2013; Shuch 2011]. IVC cross-clamp and temporary venovenous bypass are among the surgical procedures used to remove tumor thrombi of level 1 to 3 without $\mathrm{CPB}$, but in some cases we have seen unexpected and uncontrolled massive intraoperative hemorrhages. In this report, we investigate surgical management and the necessity of CPB in RCC invading the IVC.

\section{MATERIALS AND METHODS}

\section{Study Design}

We retrospectively analyzed all 15 patients who underwent nephrectomy and IVC thrombectomy at Teikyo University Hospital from May 2011 to December 2017. All patients gave informed consent, and the study protocol was approved by the ethics committee of Teikyo University. In accordance with 


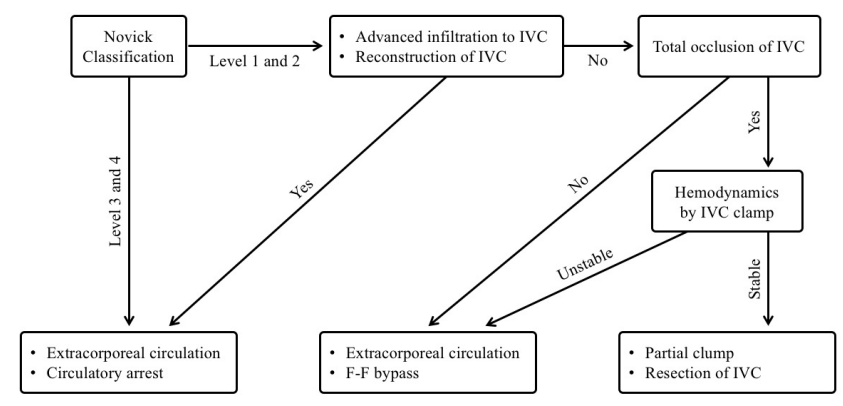

Figure 1. Surgical strategy based on Novick classification. F-F, femoralfemoral; IVC, inferior vena cava.

the American Joint Committee on Cancer Staging Manual [Neves 1987], all patients were assessed by hematologic and biochemical investigations, medical history, physical examinations, and diagnostic imaging such as abdominal Doppler ultrasonography, contrast-enhanced computed tomography, or magnetic resonance imaging. When our urologists considered additional evaluations necessary, bone scintigraphy and positron emission tomography were conducted. Novick classification was used to assess the level of tumor thrombus extension into the IVC [Blute 2004; Fuhrman 1982]: level 1, tumor thrombus involving the IVC at the level of the renal vein; level 2, infrahepatic IVC; level 3, intrahepatic IVC; and level 4, suprahepatic IVC or right atrium involvement.

To perform the complete resection of tumor thrombi, in the surgery conference, a medical team consisting of urologists, digestive surgeons, cardiovascular surgeons, and anesthesiologists discussed the classification and the size of tumor thrombus, the thickness of the IVC, and the ability to withstand circulatory dynamics by IVC cross-clamp. No mechanical circulatory support devices were used for the 7 patients without CPB. For those patients, general intraoperative parameters such as arterial pressure and respiratory rate were observed, and cross-clamp (and occasionally partial clamp) was performed for the complete resection of tumor thrombi. For patients with tumor thrombus of level 3, CPB was performed through cannulations of the common femoral artery and venous cava. In particular, where the tumor thrombus reached the junction of the hepatic vein and IVC, standard CPB techniques were used, including cannulation from the right atrium. Patients with tumor thrombus of level 4 underwent nephrectomy and IVC thrombectomy under $\mathrm{CPB}$, except for patients in whom the tumor thrombus did not significantly infiltrate the IVC wall and was removed easily. For patients with severe infiltration to the IVC wall and difficult surgical exfoliation, partial patch repair and partial reconstruction with a vascular prosthesis were performed (Figure 1).

\section{Study Variables and Operative Outcomes}

Patient characteristics, surgical procedures, and postoperative outcomes in the patients with and without $\mathrm{CPB}$ were collected. Follow-up data were obtained from clinical records in our institution. All data and variables were compared,

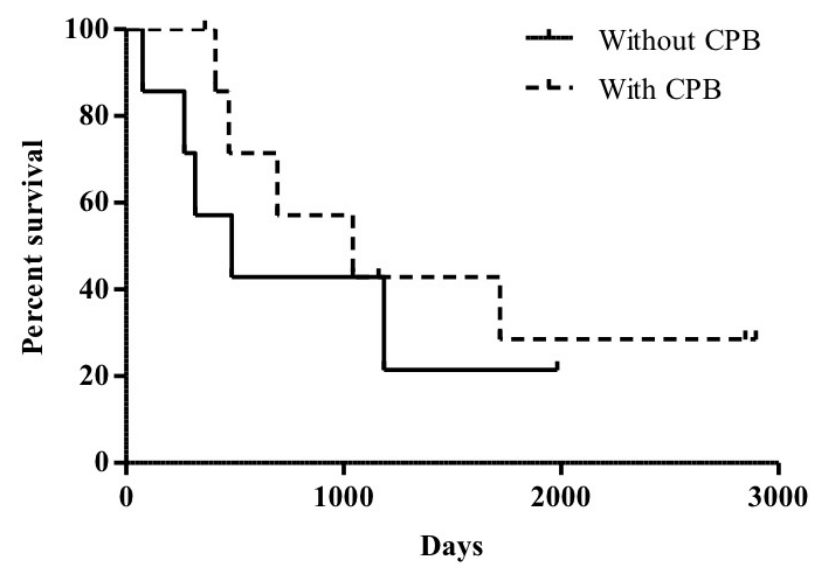

Figure 2. Kaplan-Meier estimates for all-cause survival in patients with and without cardiopulmonary bypass (CPB).

including intraoperative bleeding, operation time, postoperative coagulation function [prothrombin time (PT) and activated partial thromboplastin time (APTT)], length of stay in intensive care unit (ICU), hospitalization, postoperative metastasis, 30-day mortality, and long-term survival rates.

\section{Statistical Analysis}

Differences between the 2 groups were examined with Mann-Whitney U test for continuous variables and Fisher's exact test for categorical data. A $P$ value $<.05$ was considered statistically significant. The Kaplan-Meier method for survival analysis and the log-rank test were used. Surgical complications were classified by the Clavien-Dindo grading system [Dindo 2004].

\section{Ethical Approval}

All subjects enrolled in this research gave their informed consent, which, alongside the described protocol, has been approved by the institutional committee on human research.

\section{RESULTS}

Twelve patients were male and 3 patients were female, with an average age of $62.9 \pm 10.9$ years (range 46 to 82 ). Eleven patients had tumors on their right kidneys, and 4 patients had tumors on the left (Table 1). Novick classification of all 15 patients showed 1 patient of level 1, 1 patient of level 2, 9 patients of level 3, and 4 patients of level 4 (Table 1). The average operative times were $824 \pm 335$ minutes in the patients with $\mathrm{CPB}$ and $646 \pm 162$ minutes in the patients without CPB. The average amount of intraoperative bleeding was $2125 \pm 1315 \mathrm{ml}$ in the patients with CPB and $3333 \pm$ $1431 \mathrm{ml}$ in the patients without CPB (Table 2). For the level 3 and 4 patients, the average operative time and the average amount of intraoperative bleeding in the patients without CPB were $637 \pm 142$ minutes and $3078 \pm 732 \mathrm{ml}$, respectively. There were no significant differences in operation time and intraoperative bleeding between the groups. Indeed, the 
Table 1. Patient characteristics and surgical procedure $(n=15)^{*}$

\begin{tabular}{lc}
\hline Characteristic & Value \\
\hline Age $(y)$ & $62.9 \pm 10.9$ \\
Female sex & $3(20.0)$ \\
Affected side = right & $11(73.3)$ \\
Novick classification (all patients) & \\
1 & 1 \\
2 & 1 \\
3 & 9 \\
4 & 4 \\
Novick classification (patients with CPB) & \\
1 & $0(0)$ \\
2 & $0(0)$ \\
3 & $4(44.4)$ \\
4 & $4(100.0)$ \\
IVC technique & \\
Partial resection & 6 \\
Partial resection + RA incision & \\
Partial incision + RA incision + pancreas/spleen resection & 1 \\
Incision & 2 \\
\hline
\end{tabular}

*Data are mean \pm SD or $\mathrm{n}(\%)$ unless noted otherwise. CPB, cardiopulmonary bypass; IVC, inferior vena cava; RA, right atrium.

postoperative coagulation function of both groups had no significant difference $(\mathrm{PT}, 16.1 \pm 1.4$ seconds in the patients with $\mathrm{CPB}$ and $16.8 \pm 1.4$ seconds in the patients without $\mathrm{CPB}$, $P=.19$; APTT, $34.2 \pm 4.8$ seconds in the patients with $\mathrm{CPB}$ and $37.2 \pm 3.4$ seconds in the patients without $\mathrm{CPB}, P=.11$ ).

There was no significant difference in the average ICU length of stay or average hospitalization between the groups; however, for the level 3 and 4 patients, these variables in the patients with $\mathrm{CPB}$ were significantly different from those without CPB (Table 2). No 30-day mortality was noted. Of 15 patients, only 1 (with $\mathrm{CPB}$ ) had lung metastasis 2 years after RCC resection $(\mathrm{P}=.47)$. Follow-up was completed in all cases, with a mean follow-up time of 1061.4 days. KaplanMeier survival analysis showed no significant difference in all-cause survival between the patients with $\mathrm{CPB}$ and those without $(P=.49$; Figure 2$)$.

\section{Discussion}

Generally speaking, when discussing the use of $\mathrm{CPB}$ in patients with RCC and IVC thrombus, both intraoperative bleeding and dissemination of tumor cells should be considered [Carrascal 2008]. The most common concern among surgeons in the application of CPB seems to be increased bleeding owing to full heparinization and hypothermia during $\mathrm{CPB}$. Conversely, however, the use of $\mathrm{CPB}$
Table 2. Operative characteristics according to Novick classification*

\begin{tabular}{lccc}
\hline Characteristic & CPB & No CPB & $P$ \\
\hline All patients & $\mathrm{n}=8$ & $\mathrm{n}=7$ & \\
Operation time (min) & $824 \pm 335$ & $646 \pm 162$ & .17 \\
Bleeding (ml) & $2125 \pm 1315$ & $3333 \pm 1431$ & .14 \\
ICU length of stay (d) & $6.6 \pm 4.3$ & $3.3 \pm 2.9$ & .07 \\
Hospitalization (d) & $31.6 \pm 14.8$ & $26.5 \pm 23.7$ & .31 \\
Novick levels 3 and 4 & $\mathrm{n}=8$ & $\mathrm{n}=5$ & \\
Operation time (min) & $824 \pm 335$ & $637 \pm 142$ & .18 \\
Bleeding (ml) & $2125 \pm 1315$ & $3078 \pm 732$ & .08 \\
ICU length of stay (d) & $6.6 \pm 4.3$ & $2.0 \pm 0.8$ & .03 \\
Hospitalization (d) & $31.6 \pm 14.8$ & $15.3 \pm 3.1$ & .03 \\
\hline
\end{tabular}

*Data are mean \pm SD unless noted otherwise. CPB, cardiopulmonary bypass; ICU, intensive care unit.

can actually provide a bloodless field while facilitating tumor incision and reducing the risk of tumor embolization [Belis 2000]. Although cross-clamp, and not auxiliary devices such as CPB, seems to be sufficient for many cases of level 1 and 2 thrombi, it is necessary to consider the risk of unexpected bleeding, because actual intraoperative findings have shown infiltrations of IVC that could not be detected by CT imaging. Moreover, in patients with level 3 and 4 thrombi, the use of CPB is thought to be indispensable, because resection of the tumor and safe detachment of the severe adhesion depend on controlling intraoperative bleeding.

Regardless of Novick classification, our patients underwent $\mathrm{CPB}$ and tumor resection without severe complications. The current findings also demonstrate that ICU length of stay and hospitalization were significantly prolonged in the patients with $\mathrm{CPB}$, but there were no significant differences in coagulation function, operation time, or intraoperative bleeding with and without CPB. It is nonetheless crucial to consider the risks associated with $\mathrm{CPB}$ for each patient, because risks of up to $40 \%$ for serious complications, including renal and hepatic failure and postoperative sepsis, have been reported by some investigations [Chowdhury 2007; Magouliotis 2018].

The use of $\mathrm{CPB}$ is involved in the surgical procedures for RCC removal. Surgical options for IVC reconstruction include (1) direct repairment for small defects of IVC, (2) patch repair for large defects of IVC, and (3) graft replacement for severe invasion to the IVC wall [Dellaportas 2017]. Although direct repair is appropriate for patients with a small defect and mild invasion of IVC after RCC removal, patch repair and graft replacement with CPB are suitable in cases of severe and extensive IVC invasion to help stabilize the fluctuation of circulatory dynamics by IVC cross-clamp. Additionally, although the procedure of IVC cross-clamp should require a temporary IVC filter because several possible complications, including pulmonary embolism, are induced by 
intraoperative procedures, circulatory arrest with $\mathrm{CPB}$ may allow for the detection of remaining tumor and also prevent the occurrence of pulmonary embolism induced by floating tumor mass [Novick 1990]. Particularly, because most patients who have intraoperative acute pulmonary embolism also have high mortality, CPB may be an essential method for the prevention of unnecessary complications. Thus, of the available surgical options for the removal of RCC, the use of $\mathrm{CPB}$ should be considered.

Few studies have examined the correlation between CPB and tumor cell dissemination. The dissemination of tumor cells by CPB is a controversial subject. Some reports demonstrated that there was a low possibility of the dissemination and progression of tumor cells under CPB [Novick 1990; Langer 2016]. It is worth noting that no patients under CPB in our study so far have experienced metastasis of renal cell carcinoma. One report demonstrated that CPB could be involved in the dissemination of cancer cells by temporary immune suppression through changing the balance of cytokines, including interferon-, interleukin (IL)-10, IL-6, and IL-8 [Sablotzki 1997]. A recent retrospective cohort of 43,347 patients who underwent coronary artery bypass graft (CABG) surgery with and without $\mathrm{CPB}$ examined cancer risk and mortality [Pinto 2013]. A statistically significant increase in the risk of skin melanoma and lung cancer was observed in CABG patients with $\mathrm{CPB}$ versus those without $\mathrm{CPB}$. The current study showed that 1 patient under $\mathrm{CPB}$ had subsequent lung metastasis; however, there was no significant statistical difference between the patients with and without $\mathrm{CPB}$. Although there is no clear consensus currently whether surgical procedures with $\mathrm{CPB}$ correlate with tumor cell dissemination, further research may enable us to provide the optimal surgical strategy for patients with cancer and cardiovascular diseases.

\section{Study Limitations}

This study was subject to limitations. The decision to use $\mathrm{CPB}$ was carefully considered depending on the specificities of each patient. Moreover, the size of the study was quite small (15 patients), and the follow-up time was fairly moderate. Although surgical outcomes at our single center might not be representative of a general patient cohort, we intend to continue to investigate the benefits of this management in future cases.

\section{Conclusion}

In this study, we conclude that a surgical strategy with CPB and circulatory arrest for RCC patients can be safely performed.

\section{Acknowledgment}

We are grateful to Mr. Kento Kawai, MSc, for editorial assistance.

\section{REFERENCES}

Ali AS, Vasdev N, Shanmuganathan S, et al. 2013. The surgical management and prognosis of renal cell cancer with IVC tumor thrombus: 15 -years of experience using a multi-specialty approach at a single UK referral center. Urol Oncol 31:1298-1304.

Bachmann A, Seitz M, Graser A, et al. 2005. Tumour nephrectomy with vena cava thrombus. BJU Int 95:1373-1384.

Belis JA, Levinson ME, Pae WE Jr. 2000. Complete radical nephrectomy and vena caval thrombectomy during circulatory arrest. J Urol 163:434-436.

Blute ML, Leibovich BC, Lohse CM, et al. 2004. The Mayo Clinic experience with surgical management, complications and outcome for patients with renal cell carcinoma and venous tumour thrombus. BJU Int 94:33-41.

Carrascal Y, Gualis J, Arévalo A. 2008. Cardiac surgery with extracorporeal circulation in cancer patients: Influence on surgical morbidity and mortality and survival. Rev Esp Cardiol 61:369-375.

Chan F, Ngan Kee WD, Low JM. 2001. Anesthetic management of renal cell carcinoma with inferior vena caval extension. J Clin Anesth 13:585-587.

Chowdhury UK, Mishra AK, Seth A, et al. 2007. Novel techniques for tumor thrombectomy for renal cell carcinoma with intraatrial tumor thrombus. Ann Thorac Surg 83:1731-1736.

Ciancio G, Livingstone AS, Soloway M. 2007. Surgical management of renal cell carcinoma with tumor thrombus in the renal and inferior vena cava: The University of Miami experience in using liver transplantation techniques. Eur Urol 51:988-994.

Ciancio G, Manoharan M, Katkoori D, et al. 2010. Long-term survival in patients undergoing radical nephrectomy and inferior vena cava thrombectomy: Single-center experience. Eur Urol 57:667-672.

Ciancio G, Soloway MS. 2005. Renal cell carcinoma with tumor thrombus extending above diaphragm: avoiding cardiopulmonary bypass. Urology 66:266-270.

Dellaportas D, Arkadopoulos N, Tzanoglou I, et al. 2017. Technical intraoperative maneuvers for the management of inferior vena cava thrombus in renal cell carcinoma. Front Surg 4:48.

Dindo D, Demartines N, Clavien PA. 2004. Classification of surgical complications: A new proposal with evaluation in a cohort of 6336 patients and results of a survey. Ann Surg 240:205-213.

Dominik J, Moravek P, Zacek P, et al. 2013. Long-term survival after radical surgery for renal cell carcinoma with tumour thrombus extension into the right atrium. BJU Int 111:E59-E64.

Fuhrman SA, Lasky LC, Limas C. 1982. Prognostic significance of morphologic parameters in renal cell carcinoma. Am J Surg Pathol 6:655-663.

Janzen NK, Kim HL, Figlin RA, et al. 2003. Surveillance after radical or partial nephrectomy for localized renal cell carcinoma and management of recurrent disease. Urol Clin North Am 30:843-852.

Jemal A, Siegel R, Xu J, et al. 2010. Cancer statistics, 2010. CA Cancer $\mathrm{J}$ Clin 60:277-300.

Kaplan S, Ekici S, Do an R, et al. 2002. Surgical management of renal cell carcinoma with inferior vena cava tumor thrombus. Am J Surg 183:292-299.

Karnes RJ, Blute ML. 2008. Surgery insight: Management of renal cell carcinoma with associated inferior vena cava thrombus. Nat Clin Pract Urol 5:329-339.

Langer NB, Mercier O, Fabre D, et al. 2016. Outcomes after resection of T4 non-small cell lung cancer using cardiopulmonary bypass. Ann Thorac Surg 102:902-910. 
Lawindy SM, Kurian T, Kim T, et al. 2012. Important surgical considerations in the management of renal cell carcinoma (RCC) with inferior vena cava (IVC) tumour thrombus. BJU Int 110:926-939.

Magouliotis DE, Tasiopoulou VS, Svokos AA, et al. 2018. Extracorporeal membrane oxygenation versus cardiopulmonary bypass during lung transplantation: A meta-analysis. Gen Thorac Cardiovasc Surg 66:38-47.

Marshall FF, Dietrick DD, Baumgartner WA, et al. 1988. Surgical management of renal cell carcinoma with intracaval neoplastic extension above the hepatic veins. J Urol 139:1166-1172.

Neves RJ, Zincke H. 1987. Surgical treatment of renal cancer with vena cava extension. Br J Urol 59:390-395.

Novick AC, Kaye MC, Cosgrove DM, et al. 1990. Experience with cardiopulmonary bypass and deep hypothermic circulatory arrest in the management of retroperitoneal tumors with large vena caval thrombi.
Ann Surg 212:472-476; discussion 476-477.

Pinto CA, Marcella S, August DA, et al. 2013. Cardioplumonary bypass has a modest association with cancer progression: A retrospective cohort study. BMC Cancer 13:519.

Sablotzki A, Welters I, Lehmann N, et al. 1997. Plasma levels of immunoinhibitory cytokines interleukin-10 and transforming growth factorbeta in patients undergoing coronary artery bypass grafting. Eur J Cardiothorac Surg 11:763-768.

Shuch B, Crispen PL, Leibovich BC, et al. 2011. Cardiopulmonary bypass and renal cell carcinoma with level IV tumour thrombus: Can deep hypothermic circulatory arrest limit perioperative mortality? BJU Int 107:724-728.

Staehler G, Brkovic D. 2000. The role of radical surgery for renal cell carcinoma with extension into the vena cava. J Urol 163:1671-1675. 